\title{
Heating of Contaminants on Diamond Windows
}

\author{
Herman Bosman, Y. Y. Lau and R. M. Gilgenbach \\ University of Michigan, Ann Arbor, MI 48109-2104, USA
}

\begin{abstract}
This paper examines the RF heating of the contaminants on a diamond window. Both heating of an isolated internal graphite impurity, and of a thin film on the window surface, are discussed. Comparison with recent experiments is given. It is found that heating of isolated internal graphite impurities is unlikely to account for diamond window failure. Upon averaging over the entire window, the thin surface film in general absorbs approximately a fraction of one percent of the total incident power. Intense local heating on the surface contaminant is possible, however.
\end{abstract}

\section{INTRODUCTION}

In all RF windows, the most troublesome type of window failure occurs unexpectedly, at a power level considered "safe" in the design. For high power millimeter wave sources, such as the MW, long pulse (10 s), $140 \mathrm{GHz}$ gyrotron, even the expensive CVD diamond windows do fail occasionally.

Such failures are usually accompanied by some strong local heating on the window surface. While multipactor may cause local heating [1-3], it is unlikely to occur at frequencies above $30 \mathrm{GHz}$ [4].

In this paper, we explore some heating mechanisms in diamond windows. In particular, we examine the role of internal contaminants, such as the graphite impurities. We also examine RF absorption by a contaminant thin film on the window surface. Some recent experiments have been conducted for the latter, which we shall examine in this paper.

While the emphasis has been placed on diamond windows, the theory may be applied to windows of other types. The theory of RF absorption on an isolated contaminant may also be applied to other contexts, an example of which is shown in the Appendix.

\section{POWER ABSORPTION IN AN ISOLATED CONTAMINANT}

It is plausible that discrete contaminants inside the window material can contribute to window heating. For example, CVD diamond disks often contain dark inclusions, assumed to be amorphous carbon, up to $40 \mu \mathrm{m}$ in size $[5,6]$. These internal contaminants may have significant electrical conductivity compared with diamond. They may dissipate the RF power of the incident microwaves, causing localized heating. 
It is of interest to note that an isolated contaminant, depending on its size and its complex electrical permittivity, may absorb RF power primarily through the electric field, or primarily through the time-varying magnetic field. It turns out that an internal graphite inclusion absorbs only a small amount of RF power, primarily through the electric field. The ohmic heating of the graphite inclusion is negligible within the diamond window. [In the Appendix, using the same analysis, we show that an aluminum contaminant of $100 \mu \mathrm{m}$ size near the surface of the maximum RF magnetic field $(1 \mathrm{MA} / \mathrm{m})$ of a microwave cavity may absorb significant RF power from the timevary magnetic field to cause melting or even vaporization of the aluminum sphere.]

A single internal contaminant is modeled as a sphere of radius $a$ and electrical conductivity $\sigma$, embedded in a lossless dielectric. A plane electromagnetic wave is incident onto this contaminant sphere, with a wavelength in the dielectric $\lambda$ assumed to be much larger than both $a$ and $\delta$, where the electromagnetic skin depth $\delta=$ $\left(2 / \omega \mu_{0} \sigma\right)^{1 / 2}$. The complex permittivity of the contaminant is given by $\varepsilon+\sigma / j \omega$ and the contaminant material is assumed to be nonmagnetic, i.e. $\mu=\mu_{0}$, the free space permeability. The thermal conductivity of the dielectric material is $\kappa_{\mathrm{D}}$, and that of the contaminant is $\kappa_{\mathrm{C}}$.

In accordance with Landau and Lifshitz [7], we treat the electric- and magnetic field components of the incident microwaves separately when calculating power dissipation in the spherical contaminant. The generic expression for the power absorbed by a single contaminant $\left(P_{a b s}\right)$ is given in Equation (1), where $E$ is the incident electric field strength and $V$ is the volume of the sphere. The dimensionless polarizability $\alpha$ is a function of the particle size $a$, skin depth $\delta$ and the dielectric wavelength $\lambda$.

$$
P_{a b s}=\alpha \omega\left(1 / 2 \varepsilon E^{2}\right) V=\alpha \omega\left(1 / 2 \mu_{o} H^{2}\right) V
$$

where $H$ is the magnetic field of the incident wave.

Separate expressions for $\alpha$ are given in Landau and Lifshitz [7] depending on whether the time varying electric $\left(\alpha_{\mathrm{E}}\right)$ or magnetic $\left(\alpha_{\mathrm{H}}\right)$ field components of the incident microwaves are considered. Equations (2) and (3) give the values of $\alpha_{\mathrm{E}}$ and $\alpha_{\mathrm{H}}$ respectively in the limits of $a>>$ and $a<<\delta$.

$$
\begin{array}{lll}
\lambda>>\mathbf{a}>>\boldsymbol{\delta}: & \alpha_{E}=9 \pi^{2}\left(\frac{\delta}{a}\right)\left(\frac{a}{\lambda}\right)^{2}, & \alpha_{H}=\frac{9}{4}\left(\frac{\delta}{a}\right) \\
\lambda>>\boldsymbol{\delta}>>\mathbf{a}: & \alpha_{E}=18 \pi^{2}\left(\frac{\delta}{\lambda}\right)^{2}, & \alpha_{H}=\frac{1}{5}\left(\frac{a}{\delta}\right)^{2}
\end{array}
$$

Note from Eq. (2) that $\alpha_{H}>>\alpha_{E}$ in the limit $\lambda>>a>>\delta$. That is, in the latter limit, the dominant RF dissipation is through the eddy current generated by the time-varying magnetic field [see Appendix].

The temperature difference between the contaminant and the bulk dielectric can be calculated by solving the steady state heat conduction problem. It is again instructive to look at the two limiting cases of $a>>$ and $a<<\delta$, shown in Equations (4) and (5). 


$$
\begin{array}{cc}
\mathbf{a}>>\boldsymbol{\delta}: & \Delta T=\frac{P_{a b s}}{4 \pi a \kappa_{D}} \\
\mathbf{a}<<\boldsymbol{\delta}: & \Delta T=\frac{P_{a b s}}{8 \pi a}\left(\frac{1}{\kappa_{C}}+\frac{2}{\kappa_{D}}\right)
\end{array}
$$

In Equation (4), heat is deposited mostly in the thin skin layer on the surface of the sphere, and the heat conduction is therefore dominated by the thermal conductivity of the dielectric. In equation (5), heat is deposited more uniformly throughout the volume of the sphere, and thus the thermal conductivities of both the contaminant and the dielectric come into play.

The preceding theoretical treatment may be used to investigate the heating of a small amorphous carbon inclusion in a diamond window. We consider a $1 \mathrm{MW}$, quasi$\mathrm{CW}$ beam at $140 \mathrm{GHz}$, and assume a square beam power profile with a radius $25 \mathrm{~mm}$. The relevant physical property values used in this example are given in Figure 1.

The skin depth in this example is approximately $40 \mu \mathrm{m}$; since $\delta>a$, we may use Equations (1) and (3) to calculate the amount of power absorbed in the contaminant. The power absorbed due to electric field interactions $\left(\alpha_{E}\right)$ is about $6 \mathrm{~mW}$, whereas the power absorbed due to magnetic field interactions $\left(\alpha_{H}\right)$ is about $0.2 \mathrm{~mW}$.

In this example, a relatively low value was chosen for the thermal conductivity of the contaminant in order to get a "worst case scenario" result for the temperature increase. From Equation (5), the temperature increase is found to be only around $2^{\circ} \mathrm{C}$. It is thus concluded that heating of the internal graphite impurities is unlikely to cause diamond window failure.

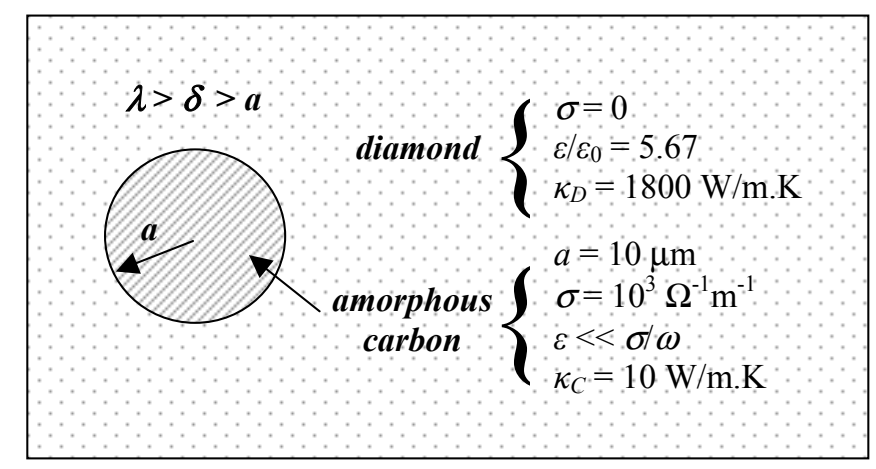

FIGURE 1. Physical model used to determine the amount of RF absorption and heating by an internal contaminant in a diamond window. 


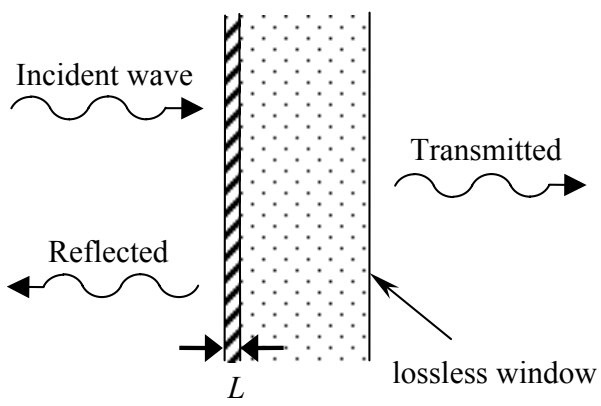

(a)

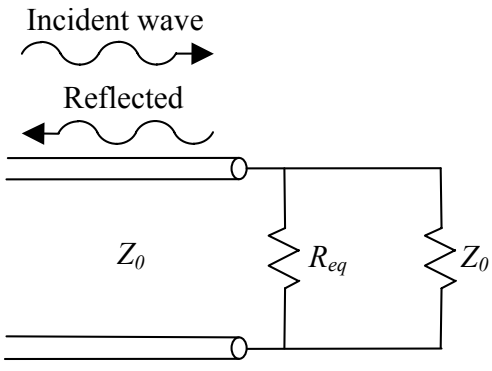

(b)

FIGURE 2. (a) A thin film on the surface of a lossless, matched window. (b) The simplified transmission line model for the thin film.

\section{POWER ABSORPTION ON THIN FILMS}

The heating of surface contaminants on RF windows is of concern, given that increased heating will increase the risk of thermal runaway and window cracking due to thermally induced stresses. Experimental investigations [8] have shown qualitatively that surface contaminants can increase the effective loss tangent of diamond windows. A recent theoretical model of microwave and millimeter wave absorption in thin films has attempted to quantify this effect [9]. Here, we apply the theory to recent measurements $[8,10]$ on diamond windows.

We consider a thin, continuous film of electrical conductivity $\sigma=\sigma(\omega)$ and thickness $L$ on the window surface, as shown in Fig. 2(a). The analysis covers two window regimes: perfectly matched windows (e.g. gyrotron diamond windows at frequencies $>100 \mathrm{GHz}$ ), and windows that are thin compared to the wavelength of the incident microwaves (e.g. klystron alumina windows at frequencies $<1 \mathrm{GHz}$ ).

The thin film is modeled as an equivalent microwave transmission line in Fig. 2(b). In the regime, $\lambda>>\delta>>L$, the thin film is modeled as a lumped resistor $R_{e q}$, given by

$$
R_{e q}=\frac{1}{\sigma L}
$$

From the transmission line model, the fraction of the incident RF power that is absorbed by the thin film $(A)$ is given by

$$
A=\frac{2 L / S}{(1+L / S)^{2}}
$$

in terms of the normalized film thickness, $L / s$. The length scale $s$ used in Equation (7) is given by

$$
s=\frac{2}{\sigma Z_{0}}, \text { hence } \frac{L}{s}=\frac{\sigma L Z_{0}}{2}=\frac{Z_{0}}{2 R_{e q}} .
$$




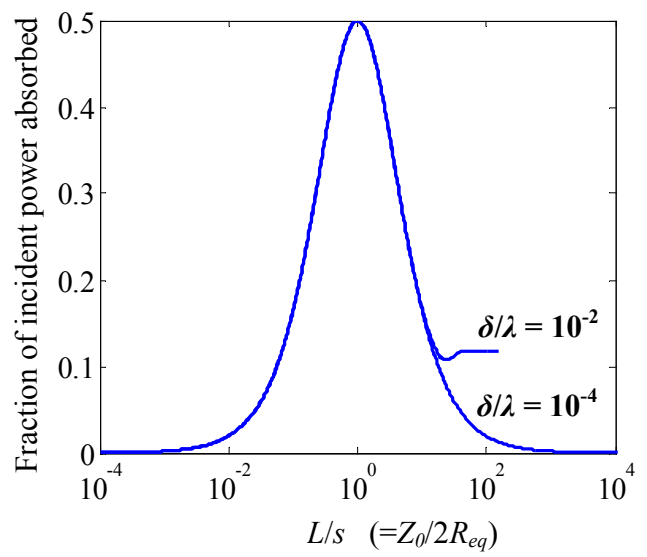

FIGURE 3. The fraction of incident power absorbed by a thin surface film on an RF window [9].

The power absorption curve in Fig. 3 was constructed from a full treatment of the thin film problem wherein Maxwell's equations were solved explicitly. In the regime where $\lambda \gg>\delta$, the curve in Fig. 3 is almost exactly described by Equation (7). It can be seen from Fig. 3 that the thin film may absorb up to $50 \%$ of the incident power, even if the film thickness $L$ is much less than the skin depth $\delta$.

The local temperature rise on a thin patch of surface contaminant has been estimated. If we take the following parameters, $\sigma=10^{3} / \Omega \mathrm{m}, \kappa=2 \mathrm{~W} / \mathrm{m}-\mathrm{K}$, for a graphite contaminant patch with imperfect crystal structures. For a thickness $L=10$ $\mu \mathrm{m}$ and radius $100 \mu \mathrm{m}$, Eqs. (6) and (8) give $R_{e q}=100 \Omega, L / s=1.9$, yielding a local absorption coefficient of about $45 \%$ from Figure 3 or Eq. (7). An incident Poynting flux of order $5 \times 10^{8} \mathrm{~W} / \mathrm{m}^{2}$ (e.g., $1 \mathrm{MW}$ over beam radius of $25 \mathrm{~mm}$ ) would lead to a maximum temperature increase of order $1200^{\circ} \mathrm{C}$.

While in the previous example we had $45 \%$ power absorption locally at a surface contaminant patch, the average or global power absorption on the window surface may be significantly different. We next compare our theory with recent measurements $[8,10]$ on diamond windows in order to estimate this global power absorption.

\section{Estimating The Thin Film Power Absorption: DC Method[10]}

A recent investigation by Jory and co-workers [10] used a DC measurement to determine $L / s$. The measurement was taken on a broken diamond gyrotron window from CPI. Two parallel silver strips, of length $w$ and gap spacing $g$ between the strips, were painted on the window surface. The resistance $R$ between the two conducting strips was measured. The resistivity $\rho$ is given by

$$
\rho=1 / \sigma=R(w L / g)
$$


where $L$ is again the thickness of the thin surface film between the silver strips. Thus, by measuring $R, w$ and $g$, Jory was able to calculate $R_{e q}$ and $L / s$ from Equations (6) and (8).

Jory [10] reported $R_{e q}$ numbers which yielded absorption values of $2 \%$ on the nucleation side of the diamond window, and $0.1 \%$ on the growth side. These numbers are to be compared with RF measurements of the loss tangents [8], as described next.

\section{Estimating The Thin Film Power Absorption: Resonator Method[8]}

Heidinger et al. [8] recently reported the results of low-power open resonator measurements on a number of diamond windows. Data were presented for the 'apparent' loss tangent over a small frequency interval. If it is assumed that the 'base' loss tangent of the window itself does not change appreciably over small frequency intervals, then the change in loss tangent is due solely to the influence of a thin film on the window surface.

An equivalent transmission line model can again be constructed to examine the influence of surface films on the resonator measurements. Figure 4 shows a window with real dielectric constant $\varepsilon$ and thickness $N(\lambda / 2)$, where $N$ is the number of halfwavelengths in the window material. As with the model used in Fig. 2(b), the surface film is modeled as the lumped resistor $R_{e q}$. The surface of the window that is in contact with the metallic mirror in the resonator is modeled as a short circuit.

The change in the 'apparent' loss tangent, relative to the 'base' window loss tangent, is given by Equation (10). This equation can be fitted to the experimental data of loss tangent $v s$. $N$ to obtain $L / s$ for a particular window.

$$
\Delta(\tan \delta)=\frac{4}{\pi}\left(\frac{L}{s}\right) \sqrt{\frac{\varepsilon_{0}}{\varepsilon}} \frac{\sin ^{2}(N \pi)}{N\left(1-\frac{\sin (2 N \pi)}{2 N \pi}\right)}, N>0.5
$$

Note that once this "global" value of $L / s$ is obtained, the average fraction $(A)$ of the power absorbed by the surface contaminants can immediately be inferred from Fig. 3.

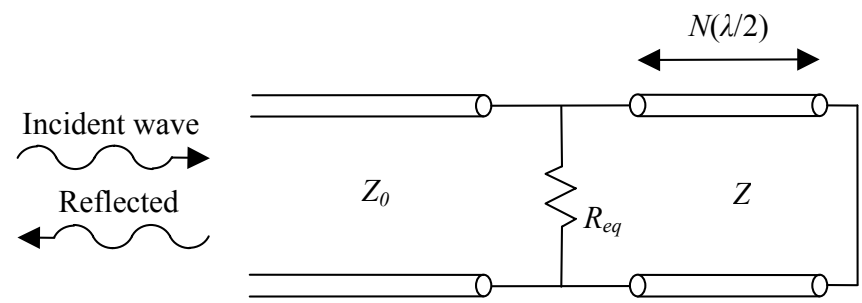

FIGURE 4. The equivalent transmission line used to model the effect of a thin surface film on the open resonator measurements of Heidinger et al. [8]. 


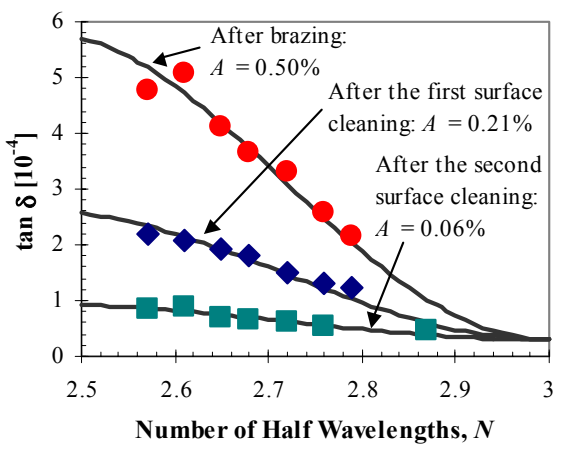

(a)

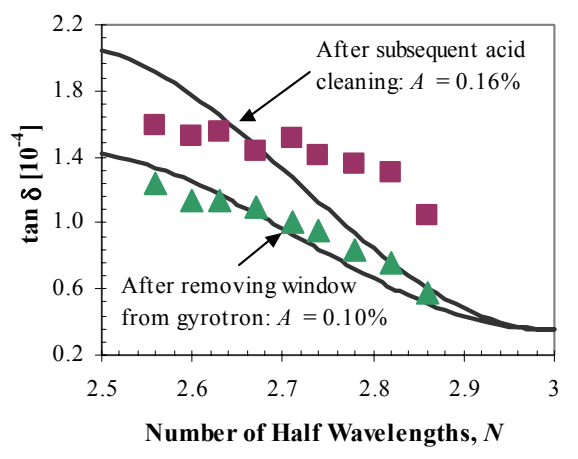

(b)

FIGURE 5. (a) Comparison of the transmission line model (solid lines) with experimental data (points) for the W7-X \#2 diamond window (growth face, from [8]), respectively after brazing (•) and

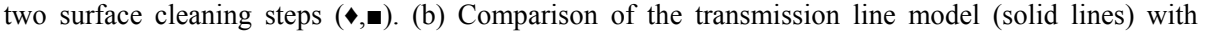
experimental data (points) for the Maquette diamond window (growth face, from [8]), after being removed from the Maquette gyrotron $(\boldsymbol{\Delta})$ and after a subsequent acid-cleaning step $(\mathbf{\square})$.

An example of this procedure is shown in Figure 5(a) for a diamond widow from the Wendelstein 7-X Stellerator program [8]. Three data sets for this particular window, designated $\mathrm{W} 7-\mathrm{X} \# 2$, were taken respectively after the gold brazing process and after two surface cleaning steps (by blasting with alumina grit). As expected, the fraction of power absorbed by the thin film $(A)$ decreases with surface cleaning, from about $0.5 \%$ to $0.06 \%$.

Figure 5(b) shows another theory-experiment comparison for the Maquette window described by Heidinger et al. [8], respectively after the window was removed from the Maquette gyrotron and after a subsequent cleaning with sulfuric acid. It is interesting to note here that the fractional power absorption value of $0.1 \%$ for the Maquette window is the same as that obtained by Jory [10] on the growth side of his diamond window using the DC method.

While the preceding analysis focused on continuous films that uniformly cover the surface of the window, in reality the surface contaminants most likely have a patchy or island structure. For example, dark patches of surface contaminants are occasionally found on diamond gyrotron windows at crystal grain boundaries $[8,10]$, and TiN coatings on alumina klystron windows can have an island structure for film thicknesses up to $4 \mathrm{~nm}$ [11]. Issues concerning patchiness of contaminants are to be examined in greater detail in the future.

\section{CONCLUSION}

Internal contaminants in a window interact with both the electric and magnetic field components of the incident microwaves. The extent of this interaction depends on the relationship between the wavelength, skin depth and contaminant size. In the case of 
diamond windows, these internal contaminants do not contribute significantly to the heating of the window.

The theoretical treatment of surface contaminants on a microwave window showed that up to $50 \%$ of the incident power on the window may be absorbed by a uniform thin film, even if the thickness of the film is much less than the skin depth [9]. For a typical diamond gyrotron window our model shows that the power absorption in the thin film can be on the order of $0.1 \%$, which is in good agreement with observed values of about $0.2 \%$.

The discrete contaminant patches on a diamond window surface can potentially heat up by as much as $1000^{\circ} \mathrm{C}$. The predicted temperature increase is however very sensitive to the contaminant thickness and electrical properties, which might explain the observation by Heidinger et al. [8] that there is only a very general correlation between light spots and the location of contaminants on the window surface.

\section{ACKNOWLEDGMENTS}

The authors wish to thank Howard Jory for many stimulating discussions, and for making available to us the results of his measurements [10]. We also wish to thank Arne Fliflet, Jeff Calame, and Dave Abe for many useful discussions. This work was supported by DOE grant DE-FG02-98ER54475, AFOSR, DUSD (S \& T) under the Innovative Microwave Vacuum Electronics MURI Program, managed by the Air Force Office of Scientific Research under Grant F49620-99-1-0297, and the Northrop Grumman Industrial Affiliates Program.

\section{APPENDIX - HEATING OF ALUMINUM CONTAMINANTS IN A MICROWAVE CAVITY}

Recent experiments at SLAC [12] found that breakdown can occur in the high magnetic field regions of waveguide-to-structure couplers. Small mushroom-shaped protrusions, associated with localized power absorption and melting, were formed on the metal surface.

We model a single protrusion as small aluminum sphere with $\sigma=3.8 \times 10^{7} \Omega^{-1} \mathrm{~m}^{-1}$ and $a=50 \mu \mathrm{m}$. The sphere is present in vacuum and is exposed to magnetic field intensities of $1 \mathrm{MA} / \mathrm{m}$ at $11.4 \mathrm{GHz}$ for pulses of around $1 \mu \mathrm{s}$. The skin depth of the sphere is about $760 \mathrm{~nm}$, which means that we may use the last term of Equation (1) and $\alpha_{H}$ from Equation (2) to estimate the amount of power absorbed in the sphere.

The thermal diffusion coefficient $D$ for aluminum is approximately $10^{-4} \mathrm{~m}^{2} / \mathrm{s}$ at room temperature, therefore it takes in the order of $\tau=a^{2} / D \approx 25 \mu$ s for the sphere to reach thermal equilibrium. Since the pulse length is much shorter than this $\tau$, we can assume that the sphere does not reach thermal equilibrium during the pulse and hence that the heat does not have enough time to diffuse out of the absorption region. The temperature increase of the sphere $(\Delta T)$ is estimated by

$$
P_{a b s} \Delta t_{p u l s e}=\rho V C \Delta T
$$


in terms of the absorbed power $P_{a b s}$, the pulse length $\Delta t_{\text {pulse }}$, sphere density $\rho(\approx 2700$ $\mathrm{kg} / \mathrm{m}^{3}$ for $\left.\mathrm{Al}\right)$ and heat capacity $C(\approx 900 \mathrm{~J} / \mathrm{kg} . \mathrm{K}$ for $\mathrm{Al})$. Since in this example the heat diffusion length scale (over the $1 \mu \mathrm{s}$ pulse) is less than the sphere radius $a$, the volume $V$ in Equation (10) is taken as the volume through which heat diffuses during the pulse.

For this example approximately $810 \mathrm{~W}$ of power is absorbed by the metal sphere. The predicted temperature increase according to Equation (11) is approximately $1000^{\circ} \mathrm{C}$, which exceeds the melting point of aluminum at $660^{\circ} \mathrm{C}$.

\section{REFERENCES}

1. D. H. Priest and R. C. Talcott, IRE Trans. Electron Devices ED-8, 243 (1961).

2. R. A. Rimmer, Ph.D. dissertation, Lancaster University, Lancaster, UK, 1988.

3. R. A. Kishek and Y. Y. Lau, Phys. Rev. Lett. 80, 193 (1998); L. K. Ang, Y. Y. Lau, R. A. Kishek and R. M. Gilgenbach, IEEE Trans. Plasma Sci. 26, 290 (1998); A. Valfells, L. K. Ang, Y. Y. Lau and R. M. Gilgenbach, Phys. Plasmas 7, 750 (2000); R. B. Anderson, W. D. Getty, M. L. Brake, Y. Y. Lau, R. M. Gilgenbach and A. Valfells, Rev. Sci. Instrum. 72, 3095 (2001).

4. A. A. Neuber, L. Laurent, Y. Y. Lau and H. Krompholz, "Windows and RF Breakdown" in HighPower Microwave Sources and Technologies, edited by R. J. Barker and E. Schamiloglu, IEEE, New York, 2001, pp. 325.

5. K. Meykens, K. Haenen, M. Nesládek, L. M. Stals, C. S. J. Pickles, and R. S. Sussmann, Diam. Rel. Mat. 9, 1021-1025 (2000).

6. R. S. Sussmann, J. R. Brandon, G. A. Scarsbrook, C. G. Sweeney, T. J. Valentine, A. J. Whitehead, and C. J. H. Wort, Diam. Rel. Mat. 3, 303-312 (1994).

7. L. D. Landau and E. M. Lifshitz, in Electrodynamics of Continuous Media, $2^{\text {nd }}$ Edition, Pergamon Press, New York, 1984, pp.322.

8. R. Heidinger, G. Dammertz, A. Meier and M. K. Thumm, IEEE Trans. Plasma Sci. 30, 800 (2002).

9. H. Bosman, Y. Y. Lau and R. M. Gilgenbach, Appl. Phys. Lett. 82, 1353 (2003).

10.H. Jory, “A Note On Resistive Films on Diamond Windows," Unpublished, 2003.

11.S. Michizono, A. Kinbara, Y. Saito, S. Yamaguchi, S. Anami and N. Matuda, J. Vac. Sci. Technol. A 10, 1180 (1992).

12. V. A. Dolgashev and S. G. Tantawi, in this Workshop. 